VERSITA

\author{
TATRA \\ MOUNTaiNS \\ Mathematical Publications \\ DOI: $10.2478 / \mathrm{v} 10127-011-0007-3$ \\ Tatra Mt. Math. Publ. 48 (2011), 73-81
}

\title{
ON TWO-SCALE CONVERGENCE AND PERIODIC UNFOLDING
}

\author{
JAN FRANCŮ
}

\begin{abstract}
Two-scale convergence is an important tool in homogenization theory. The contribution deals with various primary and adjoint (based on unfolding) approaches to the two-scale convergence and their pro-and-con.
\end{abstract}

\section{Introduction}

Two-scale convergence is an important tool in homogenization theory where convergence of the sequence of solutions $u^{\varepsilon}$ to problems, e.g.,

$$
-\operatorname{div}\left(a^{\varepsilon} \nabla u^{\varepsilon}\right)=f \quad \text { in } \Omega, \quad u^{\varepsilon}=0 \quad \text { on } \partial \Omega
$$

is studied. For a periodic bounded measurable function $a(y) \geq \alpha>0$ and sequence $\left\{\varepsilon_{n}\right\}, \varepsilon_{n} \rightarrow 0+$ (the subscript $n$ in $\varepsilon_{n}$ will be omitted) the relation $a^{\varepsilon}(x)=a\left(\frac{x}{\varepsilon}\right)$ yields a sequence of coefficients $a^{\varepsilon}$ with diminishing period $\varepsilon$. Under standard assumptions for each $\varepsilon>0$ the weak solutions $u^{\varepsilon} \in H_{0}^{1}(\Omega)$ exist, are bounded in $H^{1}(\Omega)$ and thus a subsequence $\left\{u^{\varepsilon^{\prime}}\right\}$ weakly converging in $H_{0}^{1}(\Omega)$ to a $u^{*} \in H_{0}^{1}(\Omega)$ exists. In the weak formulation of the problem

$$
\int_{\Omega} a^{\varepsilon} \nabla u^{\varepsilon} \cdot \nabla \psi \mathrm{d} x=\int_{\Omega} f v \mathrm{~d} x
$$

on the left hand side we have coefficients $a^{\varepsilon}$ and gradients $\nabla u^{\varepsilon^{\prime}}$ both weakly converging in $L^{2}(\Omega)$. Passing to the limit is not possible since the information of local oscillations are lost in the weak limit as the following counterexample shows: Both $u^{\varepsilon}(x)=v^{\varepsilon}(x)=\cos \left(\frac{x}{\varepsilon}\right)$ converge weakly to zero function, but $u^{\varepsilon}(x) v^{\varepsilon}(x)=\cos ^{2}\left(\frac{x}{\varepsilon}\right)=\frac{1}{2}\left[1+\cos \frac{2 x}{\varepsilon}\right]$ converge to the constant function $\frac{1}{2}$.

(C) 2011 Mathematical Institute, Slovak Academy of Sciences. 2010 Mathematics Subject Classification: 35B27.

Keywords: two-scale convergence, unfolding, homogenization.

This research was supported by Grant No 201/08/0874 of Czech Science Foundation. 


\section{JAN FRANCŮ}

This passing to the limit in the product of two weakly converging sequences is the crucial point in proof of convergence of the solutions $u^{\varepsilon}$. The problem was solved by construction of a special sequence of test functions in [3], in the non-periodic case by the "div-curl" lemma by M ur at and Tartar, see [12].

A straightforward solution appeared in 1989 in the so-called two-scale convergence introduced by $\mathrm{Nguetseng}$ [14] and further developed by A 1 la ir e [1]. The two-scale limit of a sequence of $x$-variable functions $\left\{u^{\varepsilon}\right\}$ is a function of variables $x, y$; in $y$ the local oscillations are conserved. For details see, e.g., survey papers [11, [10], [5].

In this paper we survey several approaches to the two-scale convergence. We start with the classical definition in Section 3, continue with dual definition based on the periodic unfolding in Section 4 and return to primary definition based on an averaging operator in Section 5. Conservation of integrals by unfolding and averaging operators (Lemma 4.4 and 5.1) is crucial. Definitions and results of Sections 4 (second half) and 5 seem to be new. The definitions are compared and fundamental properties are surveyed in Section 6 .

\section{Preliminaries}

A sequence $E=\left\{\varepsilon_{n}\right\}_{n=1}^{\infty}$ of small positive numbers $\varepsilon_{n}$ tending to zero is called scale. In this paper instead of a subscript $n \in \mathbb{Z}$ all sequences will be denoted with a superscript $\varepsilon_{n}$ from the scale $E$, but the $n$ in $\varepsilon_{n}$ will be omitted.

In the periodic homogenization $Y$ denotes the basic period in $\mathbb{R}^{N}$ called also the unit cell with points $y=\left(y_{1}, \ldots, y_{N}\right)$. It is a set having the "paving property": the space $\mathbb{R}^{N}$ can be decomposed into a countable system of disjoint $Y_{\xi}=Y+\xi$ - the cells $Y$ shifted by vectors $\xi$ from a countable set $\Xi$ in $\mathbb{R}^{N}$. The simplest case is the $N$-dimensional unit cube $Y=\langle 0,1)^{N}$ and the set of shifts $\Xi=\mathbb{Z}^{N}$. Each point $y \in \mathbb{R}^{N}$ can be decomposed into two parts: shift $[y]_{Y}$ and local position in the cell $\{y\}_{Y}$ defined by

$$
y=[y]+\{y\}, \quad \text { where } \quad[y] \in \Xi \quad \text { and } \quad\{y\} \in Y .
$$

Since $\left\{Y_{\xi} \mid \xi \in \Xi\right\}$ is pavement of $\mathbb{R}^{N}$ this decomposition is unique. In case when $Y$ is the unique cube, the decomposition (2) of a point $x$ coincides with decomposition into integer and fraction part of all coordinates of $x$.

In this paper $\Omega$ will be a bounded domain in $\mathbb{R}^{N}$, with points $x=\left(x_{1}, \ldots, x_{N}\right)$ and with a Lipschitz boundary $\partial \Omega$. For $\varepsilon>0$ we obtain $\varepsilon$-scaled paving of $\mathbb{R}^{N}$ with $\varepsilon$-scaled $\varepsilon \xi$-shifted cells $Y_{\varepsilon \xi}^{\varepsilon}=\{\varepsilon y+\varepsilon \xi \mid y \in Y\}, \xi \in \Xi$ which covers also the domain $\Omega$. Let $\Omega_{\varepsilon}$ be union of all cells $Y_{\varepsilon \xi}^{\varepsilon}$ that are subset of $\Omega$ and $\Lambda_{\varepsilon}$ be its complement in $\Omega$ containing the "uncomplete" cells in $\Omega$, i.e.,

$$
\Omega_{\varepsilon}=\left\{\bigcup Y_{\varepsilon \xi}^{\varepsilon} \mid \xi \in \Xi, Y_{\varepsilon \xi}^{\varepsilon} \subset \Omega\right\}, \quad \Lambda_{\varepsilon}=\Omega-\Omega_{\varepsilon} .
$$




\section{ON TWO-SCALE CONVERGENCE AND PERIODIC UNFOLDING}

A function $a(y)$ is said to be $Y$-periodic, if it is defined on $\mathbb{R}^{N}$ and satisfies $a(y+\xi)=a(y)$ for each $\xi \in \Xi$ and all $y \in \mathbb{R}^{N}$. In case when $Y$ is the unit cube $a(y)$ is periodic in each variable $y_{i}$ with period 1 .

Let us recall that taking a bounded measurable $Y$-periodic function $a(y)$ and the scale $E$, relation

$$
a^{\varepsilon}(x)=a\left(\frac{x}{\varepsilon}\right) \equiv a\left(\frac{x_{1}}{\varepsilon}, \ldots, \frac{x_{N}}{\varepsilon}\right)
$$

defines a sequence of periodic functions with diminishing period. It converges in $L^{p}(\Omega)$ weakly if $1 \leq p<\infty$ (weakly-* if $p=\infty$ ) to a constant function $\bar{a}$, which is the integral average of $a(y): \quad \bar{a}(x)=|Y|^{-1} \int_{Y} a(y) \mathrm{d} y$.

Function spaces of $Y$-periodic functions will be denoted by $X_{p e r}(Y)$. Its elements $a(y)$ are defined on $\mathbb{R}^{N}$ and are periodic in $y$ with period $Y$, their restriction to any bounded domain $G \subset \mathbb{R}^{N}$ is in $X(G)$, although the norm is taken over the cell $Y$ only.

\section{Classical definition}

The two-scale convergence in $L^{2}(\Omega)$ was introduced in $[14$ and further worked out in [1. Let $(p, q)$ be a pair of conjugate exponents, i.e., $p, q \in(1, \infty)$ and $\frac{1}{p}+\frac{1}{q}=1$. The classical definition, see, e.g., [1], reads as follows

Definition 3.1. A sequence of functions $u^{\varepsilon}$ in $L^{p}(\Omega)$ is said to two-scale converge to a limit $u^{0} \in L^{p}(\Omega \times Y)$ (with respect to the scale $E$ ) if

$$
\lim _{\varepsilon \rightarrow 0} \int_{\Omega} u^{\varepsilon}(x) \varphi\left(x, \frac{x}{\varepsilon}\right) \mathrm{d} x=\frac{1}{|Y|} \iint_{\Omega \times Y} u^{0}(x, y) \varphi(x, y) \mathrm{d} y \mathrm{~d} x
$$

for each test function $\varphi \in \mathcal{V}=L^{q}\left(\Omega ; C_{p e r}(Y)\right)$.

For the space $\mathcal{V}$ of admissible functions the space $C^{0}\left(\bar{\Omega}, L_{\text {per }}^{p}(Y)\right)$ can be also used. If the definition requires that the sequence $\left\{u^{\varepsilon}\right\}$ is bounded in $L^{p}(\Omega)$, then the space $\mathcal{V}=C_{0}^{\infty}(\Omega) \otimes C_{p e r}^{\infty}(Y)$ of functions with compact support in $\Omega$ is sufficient.

Although the limit can be any element of $L^{p}(\Omega \times Y)$ the space $\mathcal{V}$ of test functions in the definition cannot be the whole $L^{q}(\Omega \times Y)$, since the test function $\varphi(x, y)$ is transformed into one variable function $\varphi\left(x, \frac{x}{\varepsilon}\right)$. For integrable and therefore only measurable function $\varphi(x, y) \in L^{p}(\Omega \times Y)$ the trace $\varphi\left(x, \frac{x}{\varepsilon}\right)$ is not defined. Thus some continuity of the test functions must be added. Usually the Carathéodory conditions are assumed: measurability in one variable and continuity in the other variable. Another solution is sketched in Section 5 . 


\section{Dual definitions based on the periodic unfolding}

The alternative approach is based on the so-called periodic unfolding called also two-scale transform $\mathcal{T}_{\varepsilon}$. The idea appeared in [2]. Each function $u^{\varepsilon}$ of one variable $x$ is transformed into a function $\mathcal{T}_{\varepsilon} u^{\varepsilon}$ of both variables $x, y$. Now both $\mathcal{T}_{\varepsilon} u^{\varepsilon}$ and $u^{0}$ are in $L^{p}(\Omega \times Y)$ and the convergence can be tested in this space.

This approach removes difficulties with the space of test functions: the test function $\varphi$ can be taken from the maximal space $L^{q}\left(\Omega ; L_{p e r}^{q}(Y)\right)$. We need not take care of the space $\mathcal{V}$, admissibility and compatibility of the test functions as in the classical definition.

By means of $\varepsilon$-scaled decomposition (2) the unfolding can be defined by

$$
\left(\mathcal{T}_{\varepsilon} u\right)(x, y)=u\left(\varepsilon\left[\frac{x}{\varepsilon}\right]_{Y}+\varepsilon y\right)
$$

and the weak convergence of $\mathcal{T}_{\varepsilon} u^{\varepsilon}$ is tested in $L^{p}(\Omega \times Y)$, see [4], [13]:

Definition 4.1. The sequence $\left\{u^{\varepsilon}\right\} \subset L^{p}(\Omega)$ is said to two-scale converge to $u^{0}$ in $L^{p}(\Omega)$ with respect to the scale $E$, if $\mathcal{T}_{\varepsilon} u^{\varepsilon}$ converge to $u^{0}$ weakly in $L^{p}(\Omega \times Y)$.

This unfolding works well in case of $\Omega=\mathbb{R}^{N}$ or if the domain $\Omega$ can be written as interior of a union of "complete" cells $\varepsilon Y_{\xi}$, i.e., $\Omega_{\varepsilon}=\Omega$ and $\Lambda_{\varepsilon}=\emptyset$. Then the unfolding has an important property: it conserves integral of the functions:

$$
\int_{\Omega} u(x) \mathrm{d} x=\frac{1}{|Y|} \iint_{\Omega \times Y}\left(\mathcal{T}_{\varepsilon} u\right)(x, y) \mathrm{d} x \mathrm{~d} y .
$$

But in general the domain $\Omega$ cannot be written in this way and in $\Omega$ around its boundary $\partial \Omega$ a stripe $\Lambda_{\varepsilon}$, see (3) , of "uncomplete" cells remain. In these cells the unfolded function $\mathcal{T}_{\varepsilon} u$ is not defined, since here $\varepsilon\left[\frac{x}{\varepsilon}\right]+\varepsilon y$ overlaps $\Omega$. Also the equality (5) does not hold, namely equality in [4, Prop. 1] is not true in general case.

To remove the problem in [6] and [5] the unfolding was modified to

$$
\left(\mathcal{T}_{\varepsilon}^{0} u\right)(x, y)= \begin{cases}u\left(\varepsilon\left[\frac{x}{\varepsilon}\right]_{Y}+\varepsilon \cdot y\right) & \text { for } x \in \Omega_{\varepsilon}, \\ 0 & \text { for } x \in \Lambda_{\varepsilon} .\end{cases}
$$

The unfolding $\mathcal{T}_{\varepsilon}^{0}$ is now well defined in $\Omega$. It is used for definition of two-scale convergence for any domain $\Omega$ :

Definition 4.2. The sequence $\left\{u^{\varepsilon}\right\} \subset L^{p}(\Omega)$ is said to two-scale converge to $u^{0}$ in $L^{p}(\Omega)$ if $\mathcal{T}_{\varepsilon} u^{\varepsilon}$ defined by (6) converge to $u^{0}$ weakly in $L^{p}(\Omega \times Y)$.

This definition works for any domain $\Omega$, nevertheless the integral conservation equality (5) is lost. In [5] the problem was solved by introducing a new property: 


\section{ON TWO-SCALE CONVERGENCE AND PERIODIC UNFOLDING}

Definition 4.3. The sequence $\left\{u^{\varepsilon}\right\}$ is said to satisfy "unfolding criterion for integrals" if $\lim _{\varepsilon \rightarrow 0} \int_{\Lambda_{\varepsilon}} u^{\varepsilon}(x) \mathrm{d} x=0$.

This property requires the difference $\int_{\Omega} u^{\varepsilon}(x) \mathrm{d} x-\iint_{\Omega \times Y} \mathcal{T}_{\varepsilon}^{0}\left(u^{\varepsilon}\right)(x, y) \mathrm{d} x \mathrm{~d} y$ to go to zero, i.e., the integral conservation equality (5) holds in the limit only.

In [7, 8] and [9] a modified extension of the unfolding operator $\mathcal{T}_{\varepsilon}$ is proposed which removes the problems cited above. In the uncomplete cells $\Lambda_{\varepsilon}$ the unfolding is extended by the identity:

$$
\left(\mathcal{T}_{\varepsilon}^{*} u\right)(x, y)= \begin{cases}u\left(\varepsilon\left[\frac{x}{\varepsilon}\right]_{Y}+\varepsilon y\right) & \text { for } x \in \Omega_{\varepsilon}, \\ u(x) & \text { for } x \in \Lambda_{\varepsilon} .\end{cases}
$$

Let us survey properties of the unfolding operator $\mathcal{T}_{\varepsilon}^{*}$ :

Lemma 4.4. The unfolding $\mathcal{T}_{\varepsilon}^{*}$ defined by (7) for each $\varepsilon>0$ satisfies:

(a) The unfolding $\mathcal{T}_{\varepsilon}^{*}$ is linear and multiplicative, i.e., for $\alpha, \beta \in \mathbb{R}$

$$
\begin{gathered}
\mathcal{T}_{\varepsilon}^{*}(\alpha u+\beta v)=\alpha \mathcal{T}_{\varepsilon}^{*}(u)+\beta \mathcal{T}_{\varepsilon}^{*}(v), \\
\mathcal{T}_{\varepsilon}^{*}(u v)=\mathcal{T}_{\varepsilon}^{*}(u) \mathcal{T}_{\varepsilon}^{*}(v) .
\end{gathered}
$$

(b) The unfolding $\mathcal{T}_{\varepsilon}^{*}$ conserves the integral (up to the multiplicative constant $|Y|)$, i.e., for $f \in L^{1}(\Omega)$ there is

$$
\iint_{\Omega \times Y}\left(\mathcal{T}_{\varepsilon}^{*} f\right)(x, y) \mathrm{d} x \mathrm{~d} y=|Y| \int_{\Omega} f(x) \mathrm{d} x,
$$

which means that $\mathcal{T}_{\varepsilon}^{*}$ is an isometry (up to the multiplicative constant $\left.|Y|^{1 / p}\right)$ of spaces $L^{p}(\Omega)$ and $L^{p}(\Omega \times Y)$, i.e.,

$$
\left\|\mathcal{T}_{\varepsilon}^{*} u\right\|_{L^{p}(\Omega \times Y)}=|Y|^{\frac{1}{p}}\|u\|_{L^{p}(\Omega)} \quad \text { for } \quad u \in L^{p}(\Omega) .
$$

The unfolding $\mathcal{T}_{\varepsilon}^{*}$ enables to introduce new, "the most convenient" definition of the two-scale convergence including the strong two-scale convergence:

Definition 4.5. Let $E$ be a scale, $\left\{u^{\varepsilon}\right\}$ a sequence in $L^{p}(\Omega)$ and $u^{0} \in L^{p}(\Omega \times Y)$.

(a) The sequence $u^{\varepsilon}$ is said to (weakly) two-scale converge to $u^{0}$ in $L^{p}(\Omega)$ with respect to the scale $E$, if $\mathcal{T}_{\varepsilon}^{*} u^{\varepsilon}$ converge to $u^{0}$ weakly in $L^{p}(\Omega \times Y)$.

(b) The sequence $u^{\varepsilon}$ is said to strongly two-scale converge to $u^{0}$ in $L^{p}(\Omega)$ with respect to the scale $E$, if $\mathcal{T}_{\varepsilon}^{*} u^{\varepsilon}$ converge to $u^{0}$ strongly in $L^{p}(\Omega \times Y)$. 


\section{JAN FRANCŮ}

\section{EXAMPLE 4.6.}

(a) Let $f, g \in L^{p}(\Omega)$ and $\psi \in L^{\infty}\left(Y_{\text {per }}\right)$, s.t. $\int_{Y} \psi(y) \mathrm{d} y=0$. Then the sequence $u^{\varepsilon}(x)=f(x) \psi\left(\frac{x}{\varepsilon}\right)+g(x)$ is bounded in $L^{p}(\Omega)$. Since its unfolding $\mathcal{T}_{\varepsilon}^{*} u^{\varepsilon}$ yields $\left(\mathcal{T}_{\varepsilon}^{*} u^{\varepsilon}\right)(x, y)=f(x) \psi(y)+g(x)$ in $\Omega_{\varepsilon} \times Y, u^{\varepsilon}$ strongly two-scale converges in $L^{p}(\Omega)$ to the limit $u^{0}(x, y)=f(x) \psi(y)+g(x)$. The sequence $u^{\varepsilon}$ converges to $g(x)$ in $L^{p}(\Omega)$ weakly, but not strongly, unless $f(x) \equiv 0$ or $\psi(y) \equiv 0$. The example shows that the local oscillations of $u^{\varepsilon}$, which are lost in the usual weak $L^{p}(\Omega)$ limit, are conserved in the strong two-scale limit.

(b) In the previous example the sequence was strongly two-scale converging. It was caused by the fact that the period $\varepsilon$ of $\psi\left(\frac{x}{\varepsilon}\right)$ was "in resonance" with the scale $E=\{\varepsilon\}$. Modifying the sequence to $u^{\varepsilon}(x)=f(x) \psi\left(\frac{2 x}{\varepsilon}\right)+$ $g(x)$ with the same scale $E$, the sequence also strongly two-scale converges but the limit is $u^{0}(x, y)=f(x) \psi(2 y)+g(x)$. The weak $L^{p}(\Omega)$ limit is unchanged.

(c) If the period of the function $\psi$ is not "in resonance" with the scale $E$, i.e., their ratio is irrational, e.g., $u^{\varepsilon}(x)=f(x) \psi\left(\frac{x}{\sqrt{2} \varepsilon}\right)+g(x)$, then the sequence $\left\{u^{\varepsilon}\right\}$ does not converge two-scale strongly but only two-scale weakly. Its limit $u^{0}(x, y)=g(x)$ is independent of $y$, i.e., the local oscillations are again lost.

\section{The primary definition revisited}

In the classical Definition 3.1 the test function $\varphi(x, y)$ appears in the form $\varphi\left(x, \frac{x}{\varepsilon}\right)$, which is undefined for functions $\psi \in L^{q}(\Omega \times Y)$ and thus the space for admissible $\mathcal{V}$ should be restricted by some continuity. This restriction can be removed if the trace operator $\psi(x, y) \mapsto \psi\left(x, \frac{x}{\varepsilon}\right)$ is replaced by an averaging operator $\mathcal{U}_{\varepsilon}: L^{q}(\Omega \times Y) \rightarrow L^{q}(\Omega)$ giving the mean value of $\varphi$ over the scaled cells $Y_{\varepsilon \xi}^{\varepsilon}$. Using splitting $y=[y]_{Y}+\{y\}_{Y}$ defined be (2) it is defined by

$$
\left(\mathcal{U}_{\varepsilon} \varphi\right)(x)=\frac{1}{\left|Y^{\varepsilon}\right|} \int_{Y_{\varepsilon \xi}^{\varepsilon}} \varphi\left(t,\left\{\frac{x}{\varepsilon}\right\}_{Y}\right) \mathrm{d} t \quad \text { for } \quad x \in Y_{\varepsilon \xi}^{\varepsilon} .
$$

Also in this case problems with uncomplete cells appear: for $x \in \Lambda_{\varepsilon}$ the integral overlaps $\Omega \times Y$. To conserve isometry of the operator we modify it to:

$$
\left(\mathcal{U}_{\varepsilon}^{*} \varphi\right)(x)= \begin{cases}\left|Y^{\varepsilon}\right|^{-1} \int_{Y_{\varepsilon \xi}^{\varepsilon}} \varphi\left(t,\left\{\frac{x}{\varepsilon}\right\}_{Y}\right) \mathrm{d} t & \text { for } \quad x \in Y_{\varepsilon \xi}^{\varepsilon} \subset \Omega_{\varepsilon}, \\ |Y|^{-1} \int_{Y} \varphi(x, y) \mathrm{d} y & \text { for } \quad x \in \Lambda_{\varepsilon} .\end{cases}
$$




\section{ON TWO-SCALE CONVERGENCE AND PERIODIC UNFOLDING}

It can be easily verified that the averaging operator $\mathcal{U}_{\varepsilon}^{*}$ satisfies analogous properties as the unfolding operator $\mathcal{T}_{\varepsilon}{ }^{*}$.

Lemma 5.1. The unfolding $\mathcal{U}_{\varepsilon}^{*}$ defined by (8) for each $\varepsilon>0$ satisfies:

(a) The averaging operator $\mathcal{U}_{\varepsilon}^{*}$ is linear, i.e., for $\alpha, \beta \in \mathbb{R}$

$$
\mathcal{U}_{\varepsilon}^{*}(\alpha \varphi+\beta \psi)=\alpha \mathcal{U}_{\varepsilon}^{*}(\varphi)+\beta \mathcal{U}_{\varepsilon}^{*}(\psi) .
$$

(b) The averaging operator $\mathcal{U}_{\varepsilon}^{*}$ conserves the integrals (up to the multiplicative constant $\left.|Y|^{-1}\right)$, i.e., for $\varphi \in L^{1}(\Omega \times Y)$

$$
\int_{\Omega}\left(\mathcal{U}_{\varepsilon}^{*} \varphi\right)(x) \mathrm{d} x=\frac{1}{|Y|} \iint_{\Omega \times Y} \varphi(x, y) \mathrm{d} x \mathrm{~d} y,
$$

which means that $\mathcal{U}_{\varepsilon}^{*}$ is an isometry (up to the multiplicative constant $\left.|Y|^{-1 / p}\right)$ between $L^{q}(\Omega \times Y)$ and $L^{q}(\Omega)$, i.e.,

$$
\left\|\mathcal{U}_{\varepsilon}^{*} \varphi\right\|_{L^{p}(\Omega)}=|Y|^{-\frac{1}{p}}\|\varphi\|_{L^{p}(\Omega \times Y)} \quad \text { for } \quad \varphi \in L^{p}(\Omega \times Y) .
$$

Using the averaging operator $\mathcal{U}_{\varepsilon}^{*}$ the Definition 3.1 can be modified to:

Definition 5.2. A sequence of functions $u^{\varepsilon}$ in $L^{p}(\Omega)$ is said to two-scale converge to a limit $u^{0} \in L^{p}(\Omega \times Y)$ (with respect to the scale $E$ ) if

$$
\lim _{\varepsilon \rightarrow 0} \int_{\Omega} u^{\varepsilon}(x)\left(\mathcal{U}_{\varepsilon}^{*} \varphi\right)(x) \mathrm{d} x=\frac{1}{|Y|} \iint_{\Omega \times Y} u^{0}(x, y) \varphi(x, y) \mathrm{d} y \mathrm{~d} x
$$

for each test function $\varphi \in L^{q}(\Omega \times Y)$.

Let us remark that on contrary to Definition 3.1 in the Definition 5.2 the test function $\varphi$ is from the maximal spaces $L^{q}(\Omega \times Y)$ and the problem of the space $\mathcal{V}$ for test function disappeared.

\section{Comparison of the definitions and properties}

The unfoldings (6), (7) in Definitions 4.2, 4.5 differ in the boundary stripe $\Lambda_{\varepsilon}$ only, thus in the case when $\Lambda_{\varepsilon}$ has zero measure both definitions coincide. In case of a general domain $\Omega$ when $\left|\Lambda_{\varepsilon}\right|>0$ and $\left|\Lambda_{\varepsilon}\right| \rightarrow 0$ if the sequence $u^{\varepsilon}$ is bounded in $L^{p}(\Omega)$, then integral of $u^{\varepsilon}$ over $\Lambda_{\varepsilon}$ goes to zero and thus both definitions are equivalent.

For $Y$-periodic function $\psi$ the unfolding of $\psi_{\varepsilon}(x)=\psi\left(\frac{x}{\varepsilon}\right)$ yields in $\Omega_{\varepsilon} \times Y$

$$
\mathcal{T}_{\varepsilon} \psi_{\varepsilon}(x, y)=\psi_{\varepsilon}\left(\varepsilon\left[\frac{x}{\varepsilon}\right]_{Y}+\varepsilon y\right)=\psi\left(\left[\frac{x}{\varepsilon}\right]_{Y}+y\right)=\psi(y) .
$$




\section{JAN FRANCŮ}

For previous bounded $\psi$ and uniformly continuous $\varphi \in C^{0}(\bar{\Omega})$ the sequence of functions $\mathcal{T}_{\varepsilon}\left(\varphi \psi_{\varepsilon}\right)(x, y)$ converges uniformly to $\varphi(x) \psi(y)$ in $\bar{\Omega}_{\varepsilon} \times Y$. Using this fact one can prove that for $u^{\varepsilon}$ bounded in $L^{p}(\Omega)$ Definitions 3.1, 4.1 and 4.2 are equivalent, see, e.g., [5, Proposition 2.14]. Similarly equivalence of Definition 3.1 and Definition 5.2 can be proved.

Advantage of the Definitions 4.5 and $\left[5.2\right.$ is that unfolding operator $\mathcal{T}_{\varepsilon}^{*}$ and averaging operator $\mathcal{U}_{\varepsilon}^{*}$ conserve measure and integrals and they are isometries between corresponding spaces $L^{p}(\Omega \times Y)$ and $L^{p}(\Omega)$, see Lemma 4.4 and Lemma 5.1 . The Definition 4.5 thus yields a direct proof of principal results of the two-scale convergence theory, see [9]:

Theorem 6.1 (Compactness). Each sequence $\left\{u^{\varepsilon}\right\}_{\varepsilon \in E}$ bounded in $L^{p}(\Omega)$ contains a subsequence $\left\{u^{\varepsilon^{\prime}}\right\}_{\varepsilon^{\prime} \in E^{\prime}}$ two-scale converging to a function $u^{0} \in L^{p}(\Omega \times Y)$.

Theorem 6.2 (Passage to the limit in the product of sequences). Let $p, q, r \in(1, \infty)$ satisfy $\frac{1}{p}+\frac{1}{q}=\frac{1}{r}$ and let the sequence $\left\{u^{\varepsilon}\right\}$ converge to $u^{0}$ two-scale strongly in $L^{p}(\Omega)$ and the sequence $\left\{v^{\varepsilon}\right\}$ converge to $v^{0}$ two-scale (weakly) in $L^{q}(\Omega)$, both with respect to the same scale $E$. Then the product $u^{\varepsilon} v^{\varepsilon}$ converges to the limit $u^{0} v^{0} \equiv u^{0}(x, y) v^{0}(x, y)$ two-scale (weakly) in $L^{r}(\Omega)$.

Particularly for any $\varphi \in L^{s}(\Omega)$ with $s \in(1, \infty)$ satisfying $\frac{1}{p}+\frac{1}{q}+\frac{1}{s}=1$ :

$$
\int_{\Omega} u^{\varepsilon}(x) v^{\varepsilon}(x) \varphi(x) \mathrm{d} x \longrightarrow \frac{1}{|Y|} \iint_{\Omega \times Y} u^{0}(x, y) v^{0}(x, y) \varphi(x) \mathrm{d} x \mathrm{~d} y .
$$

Remark 6.3. The last two theorems enable to solve the problem cited in the Introduction. Since the sequence of solutions $u^{\varepsilon}$ is bounded in $H_{0}^{1}(\Omega)$, the sequence of their gradients $\left\{\nabla u^{\varepsilon}\right\}$ is bounded in $L^{2}\left(\Omega, \mathbb{R}^{N}\right)$. Due to Theorem 6.1 $\left\{\nabla u^{\varepsilon}\right\}$ contains a subsequence weakly two-scale converging to a function $\eta$. Since the coefficients are strongly two-scale converging, Theorem 6.2 enables to pass to the limit in (1), see [9].

\section{Conclusion and outline to non-periodic homogenization}

Using the modified unfolding operator we arrived to a natural definition of weak and strong two scale-convergence in Definition 4.5. Thanks to properties of the unfolding $T_{\varepsilon}^{*}$ cited in Lemma 4.4 the proofs of Theorems 6.1 and 6.2 easily follow from the theory of $L^{p}$-spaces.

The unfolding operator $\mathcal{T}_{\varepsilon}^{*}$ and averaging operator $\mathcal{U}_{\varepsilon}^{*}$ satisfying measure and integral conservation property, see Lemma 4.4 and Lemma 5.1, are starting point for general convergence for nonperiodic homogenization, see [9]. 


\section{ON TWO-SCALE CONVERGENCE AND PERIODIC UNFOLDING}

Fundamentals for theory of nonlinear homogenization was laid by Nguetseng in series of papers from 2003. In this theory the role of two-scale convergence is replaced by $\Sigma$-convergence. For citations and further information see [15].

\section{REFERENCES}

[1] ALLAIRE, G.: Homogenization and two-scale convergence, SIAM J. Math. Anal. 23 (1992), 1482-1518.

[2] ARBOGAST, T.-DOUGLAS, J.-HORNUnG, U.: Derivation of the double porosity model of single phase flow via homogenization theory, SIAM J. Math. Anal. 21 (1990), 823-836.

[3] Bensoussan, A.-LiOns, J. L.-PAPAniCOlaOU, G.: Asymptotic Analysis for Periodic Structures, in: Stud. Math. Appl., Vol. 5, North-Holland, Amsterdam, 1978.

[4] CiORAnesCU, D.-DAmlamian, A.-GRISO, G.: Periodic unfolding and homogenization, C. R. Math. Acad. Sci. Paris 335 (2002), 99-104.

[5] CIORANESCU, D.-DAMLAMIAN, A.-GRISO, G.: The periodic unfolding method in homogenization, SIAM J. Math. Anal. 40 (2008), 1585-1620.

[6] DAMLAMIAN, A.: An elementary introduction to periodic unfolding, in: Proc. of the Narvic Conference (A. Damlamian et al.), Narvik, Norway, 2004, GAKUTO Internat. Ser. Math. Sci. Appl., Vol. 24, Gakkōtosho, Tokyo, 2006, pp. 119-136.

[7] FRANCU், J.: On two-scale convergence, in: Proc. of the 6th Math. Workshop, Faculty of Civil Engineering, Brno University of Technology, Brno, October 18, 2007, CD, 7 p.

[8] FRANCŮ, J.: Modification of unfolding approach to two-scale convergence, Math. Bohem. 135 (2010), 403-412.

[9] FRANCUं, J.-SVANSTEDT, N.: Some remarks on two-scale convergence and periodic unfolding, Appl. Math., 2011 (to appear).

[10] HOLMBOM, A.-SILFVER, J.-SVANSTEDT, N.-WELLANDER, N.: On two-scale convergence and related sequential compactness topics, Appl. Math. 51 (2006), 247-262.

[11] LUKKASSEN, D.-NGUETSENG, G.-WALL, P.: Two-scale convergence, Int. J. Pure Appl. Math. 2 (2002), 35-86.

[12] MURAT, F.: Compacite par compensation, Ann. Sc. Norm. Super. Pisa Cl. Sci. (5) 22 (1978), 489-507.

[13] NECHVÁTAL, L.: Alternative approach to the two-scale convergence, Appl. Math. 49 (2004), 97-110.

[14] NGUETSENG, G.: A general convergence result for a functional related to the theory of homogenization, SIAM J. Math. Anal. 20 (1989), 608-623.

[15] NGUETSEnG, G.-SVAnSTEDT, N.: $\Sigma$-convergence. Banach J. Math. Anal. (to appear).

Received November 16, 2010

Institute of Mathematics

Faculty of Mechanical Engineering

Brno University of Technology

Technická 2

CZ-616-69 Brno

CZECH REPUBLIC

E-mail: francu@fme.vutbr.cz 